\title{
Ultrafiltrative separation of rhamnolipid from culture medium
}

\author{
Anna Witek-Krowiak · Joanna Witek • \\ Anna Gruszczyńska • Roman G. Szafran • \\ Tomasz Koźlecki · Szymon Modelski
}

Received: 28 September 2010/ Accepted: 6 January 2011/Published online: 19 January 2011

(C) The Author(s) 2011. This article is published with open access at Springerlink.com

\begin{abstract}
Classic methods of biosurfactant separation are difficult and require large amounts of organic solvents, thus generate high amounts of waste. This work presents and discusses in detail an original procedure to separate rhamnolipid from fermentation broth using high performance membrane techniques. Due to the unique properties of surface active agents, such as capability of forming aggregates above the critical micelle concentration, it is possible to easily purify the biosurfactant with high efficacy using inexpensive and commonly used membranes. In this article, two-stage ultrafiltration is proposed as a method for separating and purifying rhamnolipid from the culture medium. The obtained purified rhamnolipid solution was capable of reducing surface tension of water down to $28.6 \mathrm{mN} / \mathrm{m}$ at critical micelle concentration of $40 \mathrm{mg} / \mathrm{l}$. Separation of rhamnolipid was confirmed by HPLC; three types of rhamnolipids were identified (RL1, RL2, RL4), with considerable predominance of RL2.
\end{abstract}

Keywords Rhamnolipid · Biosurfactant · Separation · Purification - Downstream processing - Ultrafiltration . Membrane

\footnotetext{
A. Witek-Krowiak $(\bowtie) \cdot$ A. Gruszczyńska ·

R. G. Szafran · T. Koźlecki · S. Modelski

Department of Chemistry, Wrocław University of Technology, Norwida 4/6, 50-373 Wroclaw, Poland

e-mail: anna.witek@pwr.wroc.pl

J. Witek

Institute of Environment Protection Engineering, Wroclaw University of Technology, Wybrzeze Wyspianskiego 27, 50-370 Wroclaw, Poland
}

\section{Introduction}

Biosurfactants are surface active agents of amphiphilic structure, containing both hydrophilic and hydrophobic parts (Wei et al. 2005). They can be as effective as synthetic surfactants while having some clearly more favorable properties, such as lower toxicity, higher surface activity, easy production by microbial fermentation methods and biocompatibility, allowing them to be used as additives to cosmetics, pharmaceuticals and foodstuffs. However, isolation of the biosurfactant from the culture medium and production of satisfactory amounts of a pure compound continue to pose problems. Classic methods of biosurfactant separation (solvent extraction, precipitation) require large amounts of organic solvents and thus generate high amounts of waste. In order to isolate a particular component, one must sometimes use several separation methods simultaneously. This is work-consuming and expensive, and the obtained product could still be below the required purity. Therefore, there is a need for more economical and environmentally friendly separation methods.

The use of ultrafiltration membranes for biosurfactant separation was proposed in 1990 by Mulligan and Gibbs (1990) in a surfactin and rhamnolipid model. Surfactin purification method was also modified by Sen and Swaminathan (2005), who determined optimum process condition and the purity of surfactin. Lin and Jiang (1997) separated surfactin in a two-stage process utilizing membranes with different cut-off values, making use of the surfactant properties associated with formation of micellar structures. Ultrafiltration on membranes having low porosity range is perfectly suited for separation of such systems. The concentrate contained biosurfactant micelles and proteins, while the permeate contained the remaining 
medium components, i.e. sugars, dyes, mineral salts etc. Next, the concentrated retentate was diluted in appropriate alcohol (to break up the micelles) and the solution was resubmitted to ultrafiltration. As a result, the retentate contained proteins and other macromolecules, while the permeate contained a pure biosurfactant solution. Isa et al. (2007, 2008) have elaborated on the research on the aforementioned two-stage surfactin purification process. Modifications of this method were also developed by other authors (Chen et al. 2007, 2008; Juang et al. 2008), including those making that made use of the cross-flow systems (Chen et al. 2008a, b; Juang et al. 2008a, b).

In this article, two-stage method based on ultrafiltration process is proposed an analyzed as a method for separating and purifying rhamnolipid from the culture medium.

\section{Materials and methods}

Bacterial strain, culture medium and fermentation conditions

Pseudomonas aeruginosa was isolated from soil contaminated by hydrocarbons. The strain was selected for its ability to produce extracellular biosurfactants able to reduce surface tension below $40 \mathrm{mN} / \mathrm{m}$. The $P$. aeruginosa strain was identified with API ${ }^{\circledR}$ NE 20 strip (Biomérieux).

Pseudomonas aeruginosa from nutrient agar slant was inoculated into a $100 \mathrm{ml}$ Erlenmayer flask containing $50 \mathrm{ml}$ of mineral salts medium $\left(\mathrm{KH}_{2} \mathrm{PO}_{4} 1.56 \mathrm{~g} / \mathrm{l}\right.$,

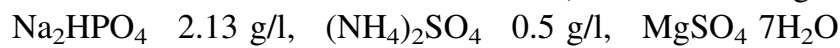
$\left.0.2 \mathrm{~g} / \mathrm{l}, \mathrm{CaCl}_{2} 2 \mathrm{H}_{2} \mathrm{O} 0.01 \mathrm{~g} / \mathrm{l}\right), 1 \mathrm{ml}$ of microelements $\left(\mathrm{FeCl}_{3} \cdot 6 \mathrm{H} 2 \mathrm{O} \quad 2,7 \mathrm{~g} / \mathrm{l}, \mathrm{H}_{3} \mathrm{BO}_{3} \quad 0,1 \mathrm{~g} / \mathrm{l}, \quad \mathrm{Ca}(\mathrm{NO} 3) 2 \cdot 6 \mathrm{H}_{2} \mathrm{O}\right.$ $0,05 \mathrm{~g} / \mathrm{l}, \quad \mathrm{ZnSO}_{4} \cdot 7 \mathrm{H}_{2} \mathrm{O} \quad 0,1 \mathrm{~g} / \mathrm{l}, \mathrm{CuSO}_{4} \cdot 5 \mathrm{H}_{2} \mathrm{O} \quad 0,005 \mathrm{~g} / \mathrm{l}$, $\left.\mathrm{MnCl}_{2} \cdot \mathrm{H}_{2} \mathrm{O} 0,005 \mathrm{~g} / \mathrm{l}\right)$ and $2 \%(\mathrm{w} / \mathrm{v})$ of glycerol as a carbon source. The inoculum was incubated for $24 \mathrm{~h}$ at $30^{\circ} \mathrm{C}$ and $133 \mathrm{rpm}$ on a rotary shaker (JW Electronic). Rhamnolipid production was carried out in a 11 flask, containing $500 \mathrm{ml}$ of mineral salts medium, $2 \%(\mathrm{w} / \mathrm{v})$ of the inoculum and $2 \%(\mathrm{w} / \mathrm{v})$ of glycerol as a carbon source and incubated as described above for 7 days. The final rhamnolipid concentration reached as much as $4000 \mathrm{mg} / \mathrm{l}$.

\section{Ultrafiltration experiments}

Separation of Pseudomonas aeruginosa from the fermentation broth was carried out on a pilot Amicon SP20 installation featuring polysulphone membranes with the cut-off value of $100 \mathrm{kDa}$ and membrane area of $0.45 \mathrm{~m}^{2}$.

A two-step ultrafiltration process was carried out using Amicon 8050 stirred cells (Millipore, USA). Several ultrafiltration membranes with molecular weight cut-offs (MWCO) of 3-50 kDa and surface areas of $13.4 \mathrm{~cm}^{2}$ were tested. Membranes made of polyethersulphone (PES, PM), regenerated cellulose (YM) were obtained from Millipore (USA) and Microdyn-Nadir (PES UP005), and membranes made of polyvinyl chloride-acrylic copolymer (XM) were obtained from Amicon Corp. (USA). The MWCOs of these membranes were: 3000 (YM3), 5000 (PES Biomax 5, PES UP005), 10,000 (PES Biomax 10), 30,000 (PM30), and 50,000 (XM50). Pressures used in this worked were in the range of $100-160 \mathrm{kPa}$.

The first ultrafiltration stage consisted in concentration of sterile medium, leading to retention of rhamnolipid micelles in the retentate. Except for micelles, the retentate contained also macromolecular compounds (mostly proteins), which had to be separated in the second stage. Subsequently, the retentate was modified by addition of methanol (as in Lin and Jiang 1997) to break up rhamnolipid micelles. This lead to the transfer of rhamnolipid molecules into the permeate in the second ultrafiltration stage, while the proteins were retained in the retentate, resulting in successful separation. In this work, the effect of the starting concentration of methanol and the amount of methanol added on the process yield was studied. At each stage, rhamnolipid concentration was determined in the feed, retentate and in permeate.

Following each series of experiments, the membranes were washed in situ with distilled water $(30 \mathrm{~min}), 0.1 \mathrm{M}$ $\mathrm{NaOH}(30 \mathrm{~min}), 0.1 \mathrm{M} \mathrm{HCl}(30 \mathrm{~min})$ and again with distilled water $(30 \mathrm{~min})$. The process was carried out under $160 \mathrm{kPa}$ at $250 \mathrm{rpm}$ and room temperature.

\section{Surface tension measurement and CMC determination}

Surface tension of serial dilutions of the biosurfactant was determined by ring method using a tensiometer at room temperature. The CMC was determined by plotting the surface tension against the concentration of rhamnolipid in the solution.

\section{Rhamnolipid quantification}

The rhamnolipid content was determined by measuring the concentration of rhamnose by the orcinol method after acidic hydrolysis of the sample (Chandrasekaran and Bemiller 1980).

\section{HPLC analysis}

Quantitative analysis of rhamnolipid was conducted by high performance liquid chromatography (HPLC) to confirm the correctness of the orcinol method results. System Summit $\times 2$ (Dionex), connected to a solvent module with a SOR-100 membrane degasser, a P680 pump. An ASI-100 autosampler, an ELSD Chromachem detector (Esa Inc.) 
and a LCQ Advantage MAX mass spectrometer (Thermo Electron Corporation) working in ESI mode was used. The source of nitrogen for the detector ELSD MS detector was a Mistral generator (Schmidlin) delivering the gas with the purity of $99.8 \%$ under pressure of 8.2 bar at $11 \mathrm{l} / \mathrm{min}$. The system operated under control of Chromeleon, version 6.7 SP3 Build 1884 software. The columns used for rhamnolipid analysis were: Zorbax SB C18 (300 ̊), $3.5 \mu \mathrm{m}$, $150 \times 4.6 \mathrm{~mm}$ (Agilent) and Chromolith $100 \times 4,6 \mathrm{~mm}$ (Merck). Water used for analysis had electrolytic conductivity below $0.06 \mathrm{mS} / \mathrm{m}$. Acetonitrile (HPLC grade) and acetic acid were obtained from POCh (Poland).

\section{Results and discussion}

The effect of membrane type on the efficacy of rhamnolipid separation at the first separation stage. Rhamnolipid retention rate was calculated from the Eq. 1.

$\% R=\left(1-\frac{C_{p}}{C_{f}}\right) \times 100$

where $\% R$-retention rate $(\%), C_{p}, C_{f}$-rhamnolipid concentrations in the permeate and the raw material, respectively $(\mathrm{mg} / \mathrm{l})$.

Rhamnolipid retention efficacy of different membranes under pressure $140 \mathrm{kPa}$ is presented in Table 1. The highest rhamnolipid retention rate was achieved for membranes with cut-offs below $10 \mathrm{kDa}$. A 5 kDa U005 Microdyn Nadir membrane was selected for further experiments due to its high retention rate of more than $90 \%$ at high yields (above $20 \mathrm{LMH}$ ).

As part of the research, the effect of the pressure on the permeate flux was studied (Fig. 1). Upon ultrafiltration of sterile medium, a significant flux drop is observed during separation, compared to pure water. This is due to increasing concentration of both micelles, and proteins at the membrane surface. Polarization concentration effect plays a crucial role in flux decline, although the permeate flux reduction may depend on different additional effects, like membrane

Table 1 The effect of MWCO on rhamnolipid retention rate and on the permeate flux

\begin{tabular}{lll}
\hline Membrane type & Retention coefficient $(\%)$ & Permeate flux (LMH) \\
\hline YM3 & 94.4 & 10.25 \\
U005 & 90.5 & 22.6 \\
Biomax 5 & 86.7 & 22.4 \\
PM10 & 85.7 & 18.8 \\
Biomax 10 & 75 & 51.9 \\
PM30 & 66.9 & 46.7 \\
XM50 & 38.1 & 68.9 \\
\hline
\end{tabular}

fouling, formation of a gel layer on the membrane surface or interactions between surfactant molecules and material of the membrane. The pressure increase leads to a slight increase in flux, and therefore, the next experiment stages were carried out under the pressure of $140 \mathrm{kPa}$.

The effect of alcohol concentration at the second stage of ultrafiltration

The concentrated retentate from the first stage (concentrated from $100 \mathrm{ml}$ down to do $5 \mathrm{ml}$ ), containing both micelles and larger proteins, was diluted with alcohol and resubmitted to ultrafiltration. Alcohol allows for breaking up the micellar structures, which enables the surfactant molecules to find their way into the permeate, allowing them to be successfully separated from proteins retained in the retentate.

Methanol concentrations of above $50 \%$ result in very successful separation, allowing for the recovery of more than $90 \%$ of rhamnolipid. The use of a more dilute alcohol (20\%) and pure water did not allow to reach such high yields, with more than half of the rhamnolipid amount being retained on the membrane. The result of the experiment supports the use of 50\% methanol, similarly to conditions used by Lin and Jiang (1997) for surfactin separation.

Dilution of the permeate from the first stage of ultrafiltration has a significant effect on the yield of rhamnolipid recovery. A fivefold dilution leads to yields largely lower than those at 1:10 dilution, while the increase in the dilution ratio to $1: 20 \mathrm{v} / \mathrm{v}$ does not have a significant effect on the biosurfactant recovery rate. The value of 1:10 v/v must be considered the optimum dilution, as higher values may lead to difficulties with evaporating the solvent in case when rhamnolipid is to be obtained in solid form.

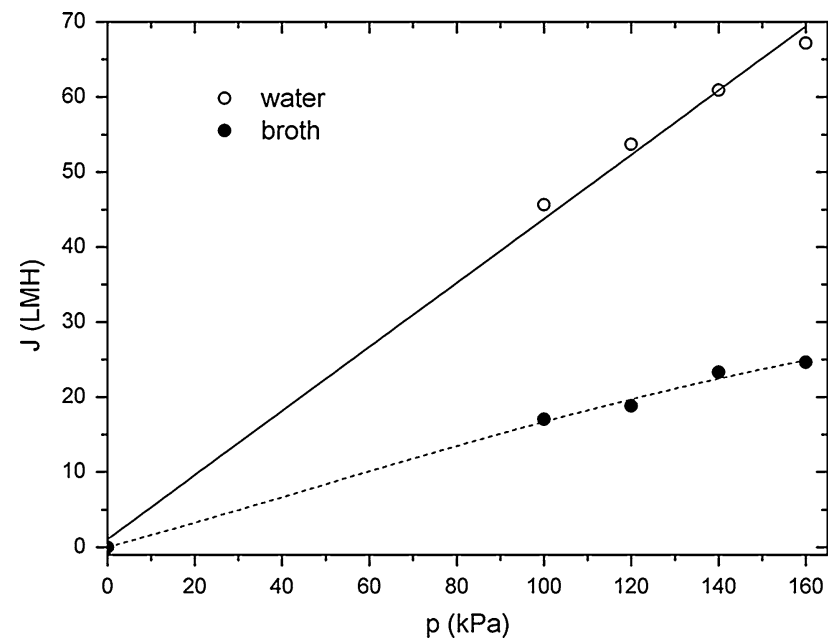

Fig. 1 The effect of pressure on rhamnolipid separation 
Fig. 2 Mass spectrum of rhamnolipid isolated by the ultrafiltration method

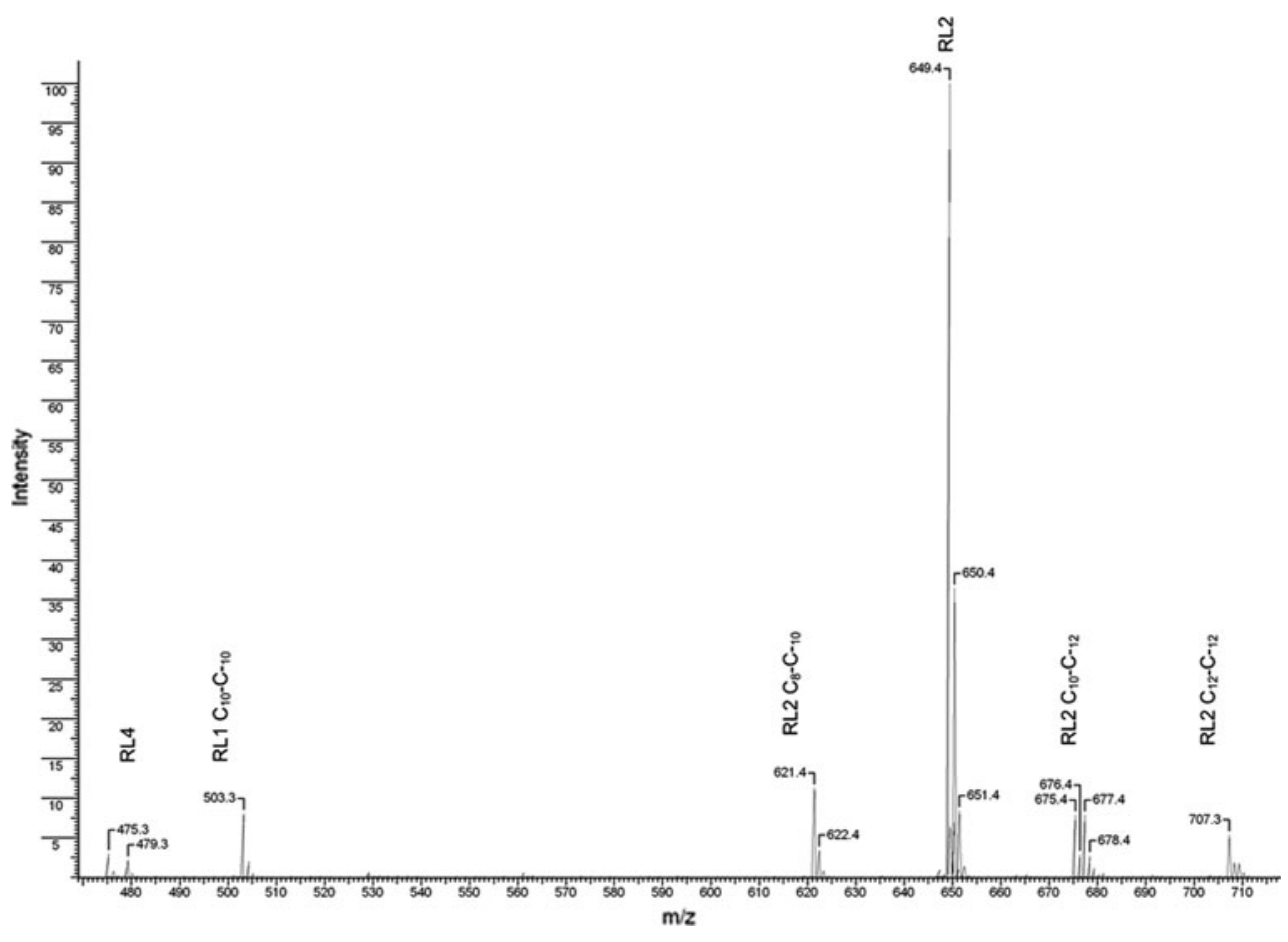

Figure 2 presents a mass spectrum of rhamnolipid solution after two-stage ultrafiltration. The spectrum confirms the presence of a series of rhamnolipid homologs in the solution, with RL2 being the predominant type, and with RL1 and RL4 also detected. The CMC of purified rhamnolipid is about $40 \mathrm{mg} / \mathrm{l}$. Rhamnolipid purified by ultrafiltration is capable of reducing the surface tension of water down to about $28.6 \mathrm{mN} / \mathrm{m}$.

One may therefore conclude that the simple two-stage ultrafiltration is an excellent alternative for the laborious physicochemical separation methods used for isolating rhamnolipid from the culture medium. The recovery of rhamnolipid in the first step of ultrafiltration was $90.5 \%$, in the second step $93.0 \%$, overall recovery in the two-stage ultrafiltration was $84.3 \%$.

\section{Conclusions}

A two-stage method of rhamnolipid purification was discussed in this work. The recovery of rhamnolipid in the two-stage ultrafiltration was $84.3 \%$. The determined CMC of the rhamnolipid was $40 \mathrm{mg} / \mathrm{l}$, and the surfactant was capable of reducing the surface tension of water down to $28.6 \mathrm{mN} / \mathrm{m}$. The method may be used both in laboratory practice, and in industrial scale applications.

Open Access This article is distributed under the terms of the Creative Commons Attribution Noncommercial License which permits any noncommercial use, distribution, and reproduction in any medium, provided the original author(s) and source are credited.

\section{References}

Chandrasekaran EV, Bemiller JN (1980) Constituent analysis of glycosaminoglycans. Methods Carbohydr Chem 8:89-96

Chen H-L, Chen Y-S, Juang R-S (2007) Separation of surfactin from fermentation broths by acid precipitation and two-stage dead-end ultrafiltration processes. J Membr Sci 299:114-121

Chen H-L, Chen Y-S, Juang R-S (2008a) Recovery of surfactin from fermentation broths by a hybrid salting-out and membrane filtration process. Sep Purif Technol 59:244-252

Chen H-L, Chen Y-S, Juang R-S (2008b) Flux decline and membrane cleaning in cross-flow ultrafiltration of treated fermentation broths for surfactin recovery. Sep Purif Technol 62:47-55

Isa MHM, Coraglia DE, Frazier RA, Jauregi P (2007) Recovery and purification of surfactin from fermentation broth by a two-step ultrafiltration process. J Membr Sci 296:51-57

Isa MHM, Frazier RA, Jauregi P (2008) A further study of the recovery and purification of surfactin from fermentation broth by membrane filtration. Sep Purif Technol 64:176-182

Juang R-S, Chen H-L, Chen Y-S (2008a) Resistance-in-series analysis in cross-flow ultrafiltration of fermentation broths of Bacillus subtilis culture. J Memb Sci 323:193-200

Juang R-S, Chen H-L, Chen Y-S (2008b) Membrane fouling and resistance analysis in dead-end ultrafiltration of Bacillus subtilis fermentation broths. Sep Purif Technol 63:531-538

Lin S-Ch, Jiang H-J (1997) Recovery and purification of the lipopeptide biosurfactant of Bacillus subtilis by ultrafiltration. Biotechnol Tech 11:413-416

Mulligan CN, Gibbs BF (1990) Recovery of biosurfactants by ultrafiltration. J Chem Technol Biotechnol 47:23-29

Sen R, Swaminathan T (2005) Characterization of concentration and purification parameters and operating conditions for the smallscale recovery of surfactin. Process Biochem 40:2953-2958

Wei QF, Mather RR, Fotheringham AF (2005) Oil removal from used sorbents using a biosurfactant. Bioresour Technol 96:331-334 